\title{
Variational Transition State Theory Calculations of Thermal Rate Coefficients for the $\mathrm{O}\left({ }^{3} \mathrm{P}\right)+\mathrm{HCl}$ Reaction
}

Thomas C. Allison ${ }^{(a)}$, B. Ramachandran ${ }^{(b)}$, Jörg Senekowitsch ${ }^{(c)}$, Donald G. Truhlar $^{(\mathrm{a})}$, and Robert E. Wyatt ${ }^{(\mathrm{d})}$

(a) Department of Chemistry and Supercomputer Institute, University of Minnesota, Minneapolis, MN 55455, USA

(b) Chemistry, College of Engineering and Science, Louisiana Tech University, Ruston, Louisiana 71272, USA

(c) College of Pharmacy, Idaho State University, Pocatello, Idaho 83209, USA

(d) Department of Chemistry and Biochemistry, University of Texas, Austin, Texas 78712, USA

\begin{abstract}
Abst ract
Variational Transition State Theory (VTST) calculations of the thermal rate coefficients of the reaction of $\mathrm{O}\left({ }^{3} \mathrm{P}\right)$ with $\mathrm{HCl}$ are presented. Four potential surfaces for the ${ }^{3} \mathrm{~A}^{\oplus}$ state are studied, three of them due to Ramachandran, Senekowitsch, and Wyatt (RSW), based on fits to scaled MR-CISD+Q energies and the fourth one due to Koizumi, Schatz, and Gordon (KSG), based on scaled MP2/6-31G $(d, p)$ energies. Using the program pol yr at e, version 7.3 , the rate coefficients are calculated using Improved Canonical Variational Theory (ICVT) with the microcanonical Optimized Multidimensional Tunneling $\left({ }^{1}\right.$ OMT) approximation over the temperature range $200 \mathrm{i} 1500 \mathrm{~K}$. These results are compared to available experimental data, which lie in the range $293 \mathrm{i} 1486 \mathrm{~K}$. It is found that the RSW surfaces yield thermal rate coefficients that are in reasonable agreement with experimental data over this range, and in very good agreement for $\mathrm{T}>500 \mathrm{~K}$, while those computed on the KSG surface are somewhat higher. These comparisons indicate that one of the RSW surfaces may establish an upper limit for the correct reaction barrier for this reaction while the KSG surface could provide a lower limit.
\end{abstract}




\section{Introduction}

The temperature dependence of thermal rate coefficients typically provides valuable information about the reaction barrier height and the contribution of tunneling to the rate at lower temperatures. The temperature dependence of the rate coefficient for the reaction of $\mathrm{O}\left({ }^{3} \mathrm{P}\right)$ with $\mathrm{HCl}$ has been studied experimentally by several workers [1-11]. These experiments have suggested values of activation energies ranging from $4.5 \mathrm{kcal} / \mathrm{mol}$ [1] to $7.5 \mathrm{kcal} / \mathrm{mol}$ [6], depending on the temperature range studied or the methods used. Theoretical investigations of this reaction prior to the early nineties were carried out using model potential surfaces constructed under the assumption that the saddle point of the triatomic system has a collinear geometry [12-15]. Brown and Smith [12], for example, used a LEPS model surface with a single semiempirical Sato parameter and a collinear saddle point with a barrier height of $7.44 \mathrm{kcal} / \mathrm{mol}$. Quasiclassical trajectory (QCT) studies on this surface yielded an activation energy of $6.6 \mathrm{kcal} / \mathrm{mol}$ over the temperature range 298 i $600 \mathrm{~K}$. Persky and Broida [13] reported the results of QCT studies on two extended LEPS surfaces where three Sato parameters were adjusted to yield good agreement between QCT rate coefficients and experimental results in the 293i $700 \mathrm{~K}$ temperature range. These two surfaces had barrier heights of 8.12 and $8.09 \mathrm{kcal} / \mathrm{mol}$, respectively, and yielded activation energies of 6.2 and $5.5 \mathrm{kcal} / \mathrm{mol}$, respectively.

In 1989, electronic structure calculations of Gordon et al. [16] indicated that the saddle point for this reaction lies at a bent geometry. However, these calculations could not provide a reliable estimate of the reaction barrier because, depending on the basis set and the level of theory used, the computed barrier heights ranged from 27.2 to $11.9 \mathrm{kcal} / \mathrm{mol}$, all of which appear to be too high. Continuing this work, Koizumi, Schatz and Gordon (KSG) obtained additional energies at the MP2/6-31G $(d, p)$ level of theory [17]. The ab initio data at this level of theory predicted a reaction barrier of $18.8 \mathrm{kcal} / \mathrm{mol}$. KSG then scaled their calculated energies to bring the approximate quantum thermal rate constant (based on the $\mathrm{J}=0$ reaction probabilities, where $\mathrm{J}$ is the total angular momentum) at $295 \mathrm{~K}$ into good agreement with the experimental value. This led to a potential energy surface on which the saddle point lies at $\underline{6} \mathrm{O}-\mathrm{H}-\mathrm{Cl}=133.4^{\circ}$ at an energy of $8.5 \mathrm{kcal} / \mathrm{mol}$ above the asymptotic reactant minimum [17]. However, more extensive quantum reactive scattering calculations by Thompson and Miller [19] as well as Nakamura and coworkers $[15,20]$ have suggested that the KSG reaction barrier may be too low.

Recently, Ramachandran, Senekowitsch, and Wyatt (RSW) calculated a new potential surface for the $\mathrm{O}+\mathrm{HCl}$ reaction which we will call Surface 1 or $\mathrm{S} 1$ [21]. This surface is based on $a b$ initio calculations at the MRCI level with the Davidson correction [22]. The Davidson corrected (MRCI+Q) energies were then scaled in a manner similar to the "scaling external correlation" (SEC) method of Brown and Truhlar [23], and fitted to a simple analytic function to yield a surface on which the saddle point has a bent geometry with $\underline{6} \mathrm{O}-\mathrm{H}-\mathrm{Cl}=132.1^{\circ}$ and a reaction barrier of $10.03 \mathrm{kcal} / \mathrm{mol}$ [24]. A QCT analysis of the reaction from the $\mathrm{HCl}$ initial states $v=2 ; j=1 ; 6 ; 9$ (where $v$ and $j$ denote the vibrational and rotational quantum numbers), at a collision energy of $3.2 \mathrm{kcal} / \mathrm{mol}$ on this surface [21] yielded $\mathrm{OH}$ rotational distributions 
and vibrational branching ratios, $\mathrm{OH}(\mathrm{v}=1)=\mathrm{OH}(\mathrm{v}=0)$, in reasonable agreement with the experimental results of Zare and coworkers [25,26].

Since that work was published, RSW computed additional points in the vicinity of the nonlinear saddle point and refitted this extended dataset. The resulting surface, hereafter called S1A, has a reaction barrier of $10.32 \mathrm{kcal} / \mathrm{mol}$. The asymptotic diatomic potentials on $\mathrm{S} 1$ and $\mathrm{S} 1 \mathrm{~A}$ are fits to scaled $a b$ initio data points which only approximately reproduce the spectroscopic constants of the molecules. In order to ensure that the potential surface accurately reproduces the known spectroscopic properties of the diatomics asymptotically, yet another surface, hereafter called S2, was obtained. The S2 surface is a fit to the same data as S1A in the three-body interaction region, but asymptotically the diatomic potentials are derived from the known spectroscopic constants of the molecules. The reaction barrier on $\mathrm{S} 2$ is $10.98 \mathrm{kcal} / \mathrm{mol}$ [27].

Therefore, at present, there are four potential surfaces available for the $\mathrm{O}\left({ }^{3} \mathrm{P}\right)+\mathrm{HCl}$ reaction on which the minimum energy path connecting the reactants to products passes through a bent transition state, as suggested by ab initio calculations. It is noteworthy that the $a b$ initio calculations that underlie the KSG and RSW surfaces represent the two mainstream approaches (i.e., single reference vs. multireference) to computational structural chemistry based on molecular orbitals. That the two approaches lead to very similar transition state geometries is encouraging. However, these surfaces vary by $2.5 \mathrm{kcal} / \mathrm{mol}$ in their barrier heights. The purpose of this paper is to use Variational Transition State Theory (VTST) [28-35] to compute thermal rate coefficients over a wide temperature range on all four surfaces and compare the results to available experimental data in order to evaluate the quality of these surfaces.

\section{Calculations}

For detailed accounts of VTST, we refer the reader to Refs. [28-34]. Below, we provide only the minimum essentials of the theory and the methods that have been used in the present set of calculations.

The VTST expression for the improved canonical variational theory (ICVT) $[28,30]$ rate constant may be written as

$$
k^{\operatorname{ICVT}}(T)=\frac{\tilde{A}}{h} k_{B} T^{\text {! }} k^{z ; 0} \min _{s}{ }^{n} \exp i \notin G^{I G T ; 0}(T ; s)=R T^{\text {i o }} ;
$$

where $k_{B}$ is Boltzmann's constant, $T$ is the temperature, $h$ is Planck's constant, $K z ; 0$ is the reciprocal of the standard state concentration, $S$ denotes a point along the minimum energy path (MEP), $\$ \mathrm{G}^{\mathrm{IGT} ; 0}(\mathrm{~T} ; \mathrm{s})$ is the standard-state improved generalized free energy of activation [30] at temperature $T$ for the generalized transition state located at $S$, and $R$ is the gas constant. Thus the VTST methods seek to minimize the calculated rate constant at a given temperature as a function of the distance $S$ along the reaction path.

To account for tunneling, the rate constant is multiplied by a transmission coefficient $\cdot{ }^{1}$ OMT , where the superscript stands for microcanonical optimized multidimensional tunneling. In this 
approximation [31], the tunneling probability at each energy is taken as the greater of those computed by the small curvature tunneling (SCT) approximation [32,33] and large curvature tunneling (LCT) approximation $[32,34]$. Thus the rate constant including the tunneling correction is written as

$$
\mathrm{k}^{\mathrm{ICVT} /{ }^{1} \mathrm{OMT}}(\mathrm{T})=\cdot{ }^{1} \mathrm{OMT}(\mathrm{T}) \mathrm{k}^{\mathrm{ICVT}}(\mathrm{T}):
$$

The calculations of $\mathrm{k}^{\mathrm{ICVT} /{ }^{1} \mathrm{OMT}}(\mathrm{T})$ reported below were performed using the pol yr at e program [36].

The $\mathrm{OHCl}$ molecule in the ${ }^{3} \mathrm{~A}^{\oplus}$ state is a nonlinear species with two degrees of bound vibrational freedom at the transition state. These two degrees of freedom correspond to the symmetric stretch and bend vibrational modes. The third vibrational degree of freedom, the asymmetric stretch, is associated with motion along the reaction path (i.e., away from the transition state region). Curvilinear internal coordinates [37] are used to both parametrize the minimum energy path and to compute the vibrational partition functions along the MEP. Anharmonicity in the vibrational coordinates are treated by the Morse I approximation [28]. Rotational partition functions are calculated classically. The electronic partition function explicitly includes the degeneracies and energy splittings of the low-lying ${ }^{3} \mathrm{P}_{2} ;{ }^{3} \mathrm{P}_{1}$, and ${ }^{3} \mathrm{P}_{0}$ spin-orbit states of reactant oxygen. A degeneracy of three is used in the transition state electronic partition function, and the $\mathrm{HCl}$ species is treated as the ground electronic state with a degeneracy of one.

In order to judge the reliability of the results presented below, we may refer to a recent survey [38] in which the ICVT/ ${ }^{1}$ OMT results were compared to accurate quantal rate coefficients. If we limit our consideration to the heavy-light-heavy mass combination, and in particular to transfers of hydrogen atoms between two non-hydrogenic atoms over the temperature interval $300 \mathrm{i} 1500$ $\mathrm{K}$, the calculations are reliable to within $27 \%$ for $\mathrm{ICVT}^{1} \mathrm{OMT}^{\mathrm{O}}$ (as compared to $397 \%$ for conventional TST). Both errors quoted are geometric means of the MUPE and LAPE percentage errors defined in the survey [38].

\section{Results and Discussion}

The saddle point characteristics of the three RSW surfaces and the KSG surface, as calculated by pol yr at e, are summarized in Table I. The $\phi \mathrm{V}_{a}^{z G}$ value reported in this table is the difference in the zero point level at the saddle point and in the asymptotic reactant valley. The saddle point location, energy, and vibrational frequencies found by pol yr at e for the KSG surface are very close to the values reported by Koizumi et al. in their original work [17]. This table shows that the RSW surfaces S1, S1A, and S2 have progressively higher reaction barriers, increasing by almost $1 \mathrm{kcal} / \mathrm{mol}$ between $\mathrm{S} 1$ and $\mathrm{S} 2$. However, due to the differences in the zero point energies for both the reactant $\mathrm{HCl}$ molecule and the saddle point $\mathrm{OHCl}$ species between the surfaces, $₫ \mathrm{~V}_{a}^{\mathrm{zG}}$ actually decreases between $\mathrm{S} 1$ and $\mathrm{S} 1 \mathrm{~A}$, and it increases only by $0.6 \mathrm{kcal} / \mathrm{mol}$ between $\mathrm{S} 1$ and $\mathrm{S} 2$. The imaginary vibrational frequency, which is associated with the asymmetric stretch mode of the transition state, also increases in going from S1 to S2. A larger imaginary frequency is associated with a more rapidly changing potential along the reaction path in the vicinity of the transition state, suggesting a "narrower" barrier, which translates into a larger tunneling correction at lower temperatures. 
In comparing the SCT and LCT tunneling calculations, the SCT tunneling probability was found to be larger than the LCT one at essentially all temperatures. In particular, for two of the surfaces, it is larger at all energies and, for the other two, it is larger at all energies $0.01 \mathrm{kcal} / \mathrm{mol}$ or more below the vibrationally adiabatic ground-state reaction barrier, $\phi \mathrm{V}_{\mathrm{a}}^{\mathrm{zG}}$.

The ICVT $/{ }^{1}$ OMT thermal rate coefficients computed using pol yr at e on the RSW potential surfaces S1, S1A, and S2 are compared with experimental results from Refs. [3], [5], [7], and [11] in Figure 1. We have excluded the experimental results of Ravisankara et al. [6] and Park [8] from this figure because the former is larger by a factor of 2 or more than other experimental results in the same temperature range, and the latter, from a shock tube study at $1050 \mathrm{~K}$, is lower by a factor of about 20 from those of [11]. It is clear from Figure 1 that the RSW surfaces yield thermal rate constants that are in reasonable agreement with experimental results at low temperature and very good agreement with experimental values for $\mathrm{T}>500 \mathrm{~K}$. Since tunneling becomes progressively less important as temperature increases, the good agreement for $\mathrm{T}>500$ $\mathrm{K}$ indicates that the reaction barriers (or activation energies) predicted by the RSW surfaces are close to the actual value. On the other hand, at lower tempertures, all three RSW surfaces predict rate coefficients that are lower than experimental observations. As one might anticipate from the comments made at the end of the preceding paragraph, surfaces S1A and S2 both show larger rate coefficients than $\mathrm{S} 1$ at low temperature, mainly due to the larger transmission coefficients .${ }^{1}$ OMT . Also shown in Figure 1 are the rate coeffecients calculated on the KSG surface using methods identical to those used for the RSW surfaces. The KSG curve lies above the majority of the experimental data points at all temperatures. This indicates that the reaction barrier of 8.5 $\mathrm{kcal} / \mathrm{mol}$ is probably too low.

Since the geometry and the real vibrational frequencies of the KSG transition state are not too different from those on the RSW surfaces, the pre-exponential factors in all four cases should be of comparable magnitude. Therefore, attributing most of the differences between the KSG and RSW curves to the exponential terms, these results suggest that the KSG surface establishes a lower limit for the reaction barrier while the S2 surface may establish an upper limit.

It is important to point out that these calculations do not include a contribution from the ${ }^{3} \mathrm{~A}^{0}$ state of the $\mathrm{OHCl}$ system, which is degenerate with the ${ }^{3} \mathrm{~A}^{\infty}$ state at asymptotic and collinear geometries, but appears to lie above it in other parts of the configuration space. The reaction barrier on the ${ }^{3} A^{0}$ surface appears to lie at a collinear geometry [39]. Based on the barrier heights on the ${ }^{3} A^{\infty}$ surfaces at collinear geometries, we conclude that this barrier is about $2 \mathrm{kcal} / \mathrm{mol}$ or more higher than the bent saddle points on the four surfaces considered here. Therefore, the contribution from the ${ }^{3} \mathrm{~A}^{0}$ state to the thermal rate constant becomes significant only at higher temperatures. If this contribution were included, the calculated rate coefficients would be slightly larger, especially for T, $1000 \mathrm{~K}$.

In order to compare the trends in the computed and experimental rate coefficients in a more quantitative fashion, we obtained fits of the computed ICVT ${ }^{1}$ OMT rate coefficients to Arrhenius-type two-parameter expressions, given by

$$
k(T)=A \exp \left(i E_{a}=R T\right) ;
$$

where the pre-exponential factor does not have a temperature dependence, and three-parameter 
fits given by

$$
k(T)=A^{9}{ }^{n} \exp (\text { i } B=T) ;
$$

where a pre-exponential temperature dependence is explicitly included. For the two-parameter fits, the lower and upper limits of the temperature intervals are used to find the unknowns $A$ and $E_{a}$. In the case of the three-parameter fits, three equations for three unknowns $A^{0}, n$, and $B$ are obtained by computing the rate coefficients at $T_{1}, T_{2}$ and $T_{3}$, where $T_{3}$ is defined as $\mathrm{T}_{3}{ }^{1}=\left(\mathrm{T}_{1}{ }^{1}+\mathrm{T}_{2}{ }^{1}\right)=2$. For the sake of comparison with experimental results, in each case, we used the same temperature interval as used by the experimentalists and the same number of parameters. We also carried out a three-parameter fit over the full 200 i $1500 \mathrm{~K}$ range that we considered theoretically.

The results of these procedures are summarized in Tables II and III. It should be noted that the parameters, particularly those for the three-parameter fits to experimental data, are extremely sensitive to the temperature ranges and the distribution of data within those ranges. They are also sensitive to the methods chosen to obtain the fits to the experimental data $\mathrm{i}$ fitting $\mathrm{k}$ vs. $\ln (\mathrm{k})$, as a function of $\mathrm{T}$ vs. $1=\mathrm{T}$, weighted vs. non-weighted least-squares, etc. $\mathrm{i}$ which may vary from one group to another. Therefore, it is not meaningful to directly compare the parameters obtained by experimentalists with those obtained in this work. It is more meaningful to compare the computed rate constants from each fit, particularly at the $T_{3}$ for the temperature range considered. Such a comparison is presented in Table IV for both the two- and the threeparameter fits. The quantity " in this table is the geometric mean of the percent errors " for a given surface, defined as

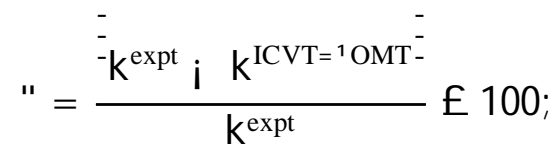

where $k^{\text {expt }}$ is calculated at $T_{3}$ from the experimental fit. Figure 1 clearly shows that the rate coefficients computed for the $\mathrm{S} 2$ surface yield the temperature dependence that best approximates the experimental data. The geometric mean of the percent errors in Table IV also support this conclusion.

Approximate quantum mechanical thermal rate coefficients for this reaction on the KSG surface, computed recently by Thompson and Miller [40] and by Poirier [41], using J -shifting and the centrifugal sudden (or helicity-conserving) approximations, are in good agreement with the present $\mathrm{ICVT} /{ }^{1} \mathrm{OMT}$ results in the $300 \mathrm{i} 500 \mathrm{~K}$ temperature range. The geometric mean of the percent deviations \pm defined as

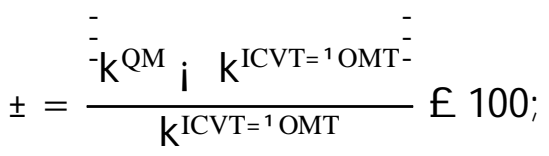

at $300,350,400$, and $500 \mathrm{~K}$ is $20.9 \%$ for the results of Ref. [40] and $15.2 \%$ for those of Ref. [41]. The quantal rate coefficients are larger than the ICVT/ ${ }^{1}$ OMT results for $\mathrm{T}<350$ $\mathrm{K}$ (by as much as \pm ' $178 \%$ at $200 \mathrm{~K}$ ) whereas the reverse is true for higher temperatures ( \pm ' $63 \%$ for Ref. [40] and ' $30 \%$ for Ref. [41] at $700 \mathrm{~K}$ ). The fact that the low temperature quantal rate coefficients are larger than those of this work is quite possibly an indication that the ${ }^{1}$ OMT method is underestimating the amount of tunneling permitted by the KSG surface. This suggests that the RSW surfaces, especially the S2 surface, could yield rate coefficients that 
are in somewhat better agreement with experimental results at low temperatures than suggested by the present results. However, this is not definitive, because the quantum calculations are approximate.

Thermal rate coefficients are, of course, the most highly averaged dynamical quantities for a chemical reaction. While the temperature dependence of the rate coefficient can yield very useful information about the barrier height and the activation energy for a reaction, only accurate dynamical calculations that yield less averaged quantities such as state-to-state reaction probabilities can provide a reliable measure for the accuracy of whole potential surfaces. Quasiclassical trajectory calculations on the three RSW surfaces presented here are underway [27]. Detailed QCT calculations on the KSG surface are also nearing completion [42]. The comparisons of the results of these calculations to state-resolved experimental data, such as the state-to-state cross section measurements of the Zare group [25,26], will help to determine whether any of these four potential surfaces can completely account for the dynamics of the $\mathrm{O}\left({ }^{3} \mathrm{P}\right)+\mathrm{HCl}$ reaction.

\section{Acknowledgments}

This research is supported by a grant to BR from the Louisiana Education Quality Support Fund, under contract no. LEQSF (1994-97)-RD-A-18. REW acknowledges support from the National Science Foundation and the Welch Foundation, and DGT acknowledges support from the U.S. Department of Energy, Office of Basic Energy Sciences. We are grateful to Drs. Javier Aoiz and Juan E. Verdasco for sending us a subroutine to evaluate the KSG potential surface and its derivatives, and to Dr. B. Poirier for sending us the quantal rate coefficients from Refs. [40] and [41].

\section{References}

[1] V. P. Balakhnin, V. I. Egorov, and E. I. Intezarova, Kinet. Katal. 12 (1971) 299; Kinet. Catal. 12 (1971) 258.

[2] E. L. Wong and F. E. Belles, NASA Tech. Note D-6495 (1975); Chem. Abstr. 76 (1972) $18326 \mathrm{q}$.

[3] D. Singleton and R. Cvetanovic, Int. J. Chem. Kinet. 13 (1975) 301.

[4] R. D. H. Brown, G. P. Glass, and I. W. M. Smith, Chem. Phys. Lett. 32 (1975) 517.

[5] R. D. H. Brown, and I. W. M. Smith, Int. J. Chem. Kinet. 7 (1975) 301.

[6] A. R. Ravisankara, G. Smith, R. T. Watson, and D. D. Davis, J. Phys. Chem. 81 (1977) 2220.

[7] W. Hack, G. Mex, and H. G. Wagner, Ber. Bunsenges. Phys. Chem. 81 (1977) 677.

[8] C. Park, J. Phys. Chem. 81 (1977) 499.

[9] D. L. Baulch, J. Duxbury, S. J. Grant, and D. C. Montague, J. Phys. Chem. Ref. Data 10 Supp. 1 (1981). 
[10] D. L. Baulch, R. A. Cox, R. F. Hampton, Jr., J. A. Kerr, J. Troe, and R. T. Watson, J. Phys. Chem. Ref. Data 13 (1984) 1259.

[11] K. Mahmud, J. S. Kim, and A. Fontijn, J. Phys. Chem. 94 (1990) 2994.

[12] R. D. Brown and I. W. M. Smith, Int. J. Chem. Kinet. 10 (1978) 1.

[13] A. Persky and M. Broida, J. Chem. Phys. 81 (1984) 4352.

[14] H. Koizumi and G. C. Schatz, Int. J. Quant. Chem. 23 (1989) 137; in Advances in Molecular Vibration and Collision Dynamics, J. M. Bowman and M. A. Ratner, Eds., JAI, Greenwitch, CT, 1990.

[15] S. C. Park, H. Nakamura, and A. Ohsaki, J. Chem. Phys. 92 (1990) 6538.

[16] M. S. Gordon, K. K. Baldridge, D. E. Bernholdt, and R. J. Bartlett, Chem. Phys. Lett. 158 (1989) 189.

[17] H. Koizumi, G. C. Schatz and M. S. Gordon, J. Chem. Phys. 95 (1991) 6421.

[18] K. Moribayashi and H. Nakamura, J. Phys. Chem. 99 (1995) 15410.

[19] W. H. Thompson and W. H. Miller, J. Chem. Phys. 106 (1997) 142.

[20] K. Nobusada, K. Moribayashi, and H. Nakamura, J. Chem. Soc. Faraday Trans. 93 (1997) 721.

[21] B. Ramachandran, J. Senekowitsch, and R. E. Wyatt, Chem. Phys. Lett. 270 (1997) 387.

[22] S. R. Langhoff and E. R. Davidson, Int. J. Quant. Chem. 8 (1974) 61.

[23] F.B. Brown and D. G. Truhlar, Chem. Phys. Lett. 117 (1985) 307.

[24] These numbers, which are slightly different from those reported in [21], result from a more accurate determination of the saddle point properties.

[25] R. J. Rakestraw, K. G. McKendrick, and R. N. Zare, J. Chem. Phys. 87 (1987) 7341.

[26] R. Zhang, W. J. van der Zande, M. J. Bronikowski, and R. N. Zare, J. Chem. Phys. 94 (1991) 2704.

[27] B. Ramachandran, J. Senekowitsch, and R. E. Wyatt, Chem. Phys. Lett., to be submitted.

[28] A. D. Issacson and D. G. Truhlar, J. Chem. Phys. 76 (1982) 1380; D. G. Truhlar, A. D. Issacson, and B. C. Garrett, in Theory of Chemical Reaction Dynamics, M. Baer, Ed., CRC Press, Boca Raton, FL, 1985; p. 65.

[29] B. C. Garrett and D. G. Truhlar, J. Phys. Chem. 83 (1979) 1052, 1079; S. C. Tucker and D. G. Truhlar in New Theoretical Concepts for Understanding Organic Reactions, J. Bertrán and I. G. Csizmadia, Eds., NATO ASI Series C, Vol. 267, Kluwer, Dordrecht, The Netherlands, 1989; p. 291.

[30] B. C. Garrett, D. G. Truhlar, R. S. Grev, and A. W. Magnuson, J. Phys. Chem. 84 (1980) 1730; 87 (1983) 4554(E).

[31] Y.-P. Liu, D.-h. Lu, A. Gonzàlez-Lafont, D. G. Truhlar, and B. C. Garrett, J. Am. Chem. Soc. 115 (1993) 7806.

[32] D.-h. Lu, T. N. Truong, V. S. Melissas, G. C. Lynch, Y.-P. Liu, B. C. Garrett, R. Steckler, A. D. Issacson, S. N. Rai, G. C. Hancock, J. G. Lauderdale, T. Joseph, and D. G. Truhlar, Comp. Phys. Comm. 71 (1992) 235.

[33] Y.-P. Liu, G. C. Lynch, T. N. Truong, D.-h. Lu, and D. G. Truhlar, J. Am. Chem. Soc. 115 
(1993) 2408.

[34] T. N. Truong, D.-h. Lu, G. C. Lynch, Y.-P. Liu, V. S. Melissas, J. J. P. Stewart, R. Steckler, B.C. Garrett, A. D. Issacson, A. Gonzàlez-Lafont, S. N. Rai, G. C. Hancock, T. Joseph, and D. G. Truhlar, Comp. Phys. Comm. 75 (1993) 143.

[35] D. G. Truhlar, B. C. Garrett, and S. J. Klippenstein, J. Phys. Chem. 100 (1996) 12771.

[36] R. Steckler, Y.-Y. Chuang, P. L. Fast, E. L. Coitiño, J. C. Corchado, W.-P. Hu, Y.-P. Liu, G. C. Lynch, K. A. Nguyen, C. F. Jackels, M. Z. Gu, I. Rossi, S. Clayton, V. S. Melissas, B. C. Garrett, A. D. Issacson, and D. G. Truhlar, pol yr at e, version 7.3.1, University of Minnesota, Minneapolis, 1997. The latest version of pol yr at e may be obtained at ht t p: / / comp. chem. umn. edu/pol yrat e.

[37] C. F. Jackels, Z. Gu, and D. G. Truhlar, J. Chem. Phys. 102 (1995) 3188.

[38] T. C. Allison and D. G. Truhlar, in Modern Methods for Multidimensional Dynamics Computations in Chemistry, D. L. Thompson, Ed., World Scientific, Singapore, in press.

[39] J. Senekowitsch, unpublished calculations.

[40] W. H. Thompson and W. H. Miller, J. Chem. Phys. 107 (1997) 2164.

[41] B. Poirier, J. Chem. Phys. (to be published).

[42] J. Aoiz, private communication. 


\begin{tabular}{|c|c|c|c|c|}
\hline $\begin{array}{l}\text { Table I. Saddle point properties of the potential } \\
\text { energy surfaces used in this study. }\end{array}$ & & & & \\
\hline & $\mathrm{S} 1$ & S1A & $\mathrm{S} 2$ & KSG \\
\hline $\mathrm{R}_{\mathrm{HCl}}^{\mathrm{z}}\left(\mathrm{a}_{0}\right)$ & 2.712 & 2.687 & 2.691 & 2.620 \\
\hline $\mathrm{R}_{\mathrm{OH}}^{\mathrm{z}}\left(\mathrm{a}_{0}\right)$ & 2.372 & 2.382 & 2.394 & 2.328 \\
\hline$\mu_{\mathrm{OHCl}}^{\mathrm{z}}$ (deg:) & 132.1 & 134.6 & 132.9 & 133.4 \\
\hline$!{ }_{1}^{\mathrm{z}}\left(\mathrm{cm}^{\mathrm{i}}\right)$ & 1446 & 1395 & 1408 & 1332 \\
\hline$!_{2}^{z}\left(\mathrm{~cm}^{\mathrm{i}^{1}}\right)$ & 316 & 309 & 267 & 429 \\
\hline$!_{\mathrm{F}}^{\mathrm{Z}}\left(\mathrm{cm}^{\mathrm{i}^{1}}\right)$ & $1537 i$ & $1603 i$ & $2042 \mathrm{i}$ & $1337 i$ \\
\hline$\phi \mathrm{V}^{\mathrm{z}}(\mathrm{kcal} / \mathrm{mol})$ & 10.03 & 10.32 & 10.98 & 8.50 \\
\hline$\phi \mathrm{V}_{\mathrm{a}}^{\mathrm{ZG}}(\mathrm{kcal} / \mathrm{mol})$ & 8.47 & 8.30 & 9.10 & 6.75 \\
\hline
\end{tabular}

\begin{tabular}{|c|c|c|c|}
\hline \multicolumn{4}{|c|}{ Table II. Parameters for two-parameter fits to rate coefficien } \\
\hline $\mathrm{T}_{1}(\mathrm{~K})$ & 298 & 293 & 293 \\
\hline $\mathrm{T}_{2}(\mathrm{~K})$ & 360 & 440 & 718 \\
\hline$T_{3}(\mathrm{~K})$ & 326 & 352 & 416 \\
\hline \multicolumn{4}{|c|}{ S1 surface } \\
\hline$A\left(10^{i 11} \mathrm{~cm}^{3} \mathrm{molec}^{i 1} \sec ^{i 1}\right)$ & $0: 891$ & $1: 10$ & 1:91 \\
\hline $\mathrm{E}_{\mathrm{a}}(\mathrm{kcal} / \mathrm{mol})$ & $7: 72$ & $7: 84$ & 8:16 \\
\hline \multicolumn{4}{|c|}{ S1A surface } \\
\hline$A\left(10^{i 11} \mathrm{~cm}^{3}\right.$ molec $\left.^{i}{ }^{1} \sec ^{i}{ }^{1}\right)$ & $0: 677$ & 0:874 & $1: 69$ \\
\hline $\mathrm{E}_{\mathrm{a}}(\mathrm{kcal} / \mathrm{mol})$ & $7: 30$ & $7: 45$ & 7:83 \\
\hline \multicolumn{4}{|c|}{ S2 surface } \\
\hline$A\left(10^{i 11} \mathrm{~cm}^{3}\right.$ molec $\left.^{i}{ }^{1} \sec ^{i}\right)$ & $0: 182$ & $0: 301$ & 0:929 \\
\hline $\mathrm{E}_{\mathrm{a}}(\mathrm{kcal} / \mathrm{mol})$ & 6:13 & $6: 42$ & 7:08 \\
\hline \multicolumn{4}{|c|}{ KSG surface } \\
\hline$A\left(10^{i 11} \mathrm{~cm}^{3}\right.$ molec $\left.^{i}{ }^{1} \sec ^{i}{ }^{1}\right)$ & $0: 681$ & $0: 844$ & 1:41 \\
\hline $\mathrm{E}_{\mathrm{a}}(\mathrm{kcal} / \mathrm{mol})$ & 6:11 & $6: 23$ & 6:53 \\
\hline \multicolumn{4}{|c|}{ Fits to experimental results } \\
\hline Reference & [3] & [5] & [7] \\
\hline$A\left(10^{i}{ }^{11} \mathrm{~cm}^{3} \mathrm{molec}^{i}{ }^{1} \mathrm{sec}^{i}{ }^{1}\right)$ & $0: 508$ & $0: 25$ & $0: 863$ \\
\hline $\mathrm{E}_{\mathrm{a}}(\mathrm{kcal} / \mathrm{mol})$ & $6: 28$ & $5: 9$ & $6: 42$ \\
\hline
\end{tabular}




\begin{tabular}{|c|c|c|c|}
\hline \multicolumn{4}{|c|}{ Table III. Parameters for three-parameter fits to rate coefficients. } \\
\hline $\mathrm{T}_{1}(\mathrm{~K})$ & 200 & 353 & 350 \\
\hline $\mathrm{T}_{2}(\mathrm{~K})$ & 1500 & 1486 & 1480 \\
\hline$T_{3}(\mathrm{~K})$ & 353 & 570 & 566 \\
\hline \multicolumn{4}{|c|}{ S1 surface } \\
\hline$A^{0}\left(10^{i}{ }^{16} \mathrm{~cm}^{3} \mathrm{molec}^{i^{1}} \mathrm{sec}^{i^{1}}\right)$ & $0: 144$ & 1:70 & $1: 43$ \\
\hline $\mathrm{n}$ & 1:94 & $1: 62$ & $1: 64$ \\
\hline $\mathrm{B}(\mathrm{K})$ & 3188 & 3401 & 3385 \\
\hline \multicolumn{4}{|c|}{ S1A surface } \\
\hline$A^{0}\left(10^{i}{ }^{16} \mathrm{~cm}^{3} \mathrm{molec}^{i 1} \mathrm{sec}^{i^{1}}\right)$ & $6: 94 f 10^{i 3}$ & 0:402 & $0: 396$ \\
\hline $\mathrm{n}$ & $2: 32$ & $1: 80$ & $1: 80$ \\
\hline$B(\mathrm{~K})$ & 2806 & 3156 & 3157 \\
\hline \multicolumn{4}{|c|}{ S2 surface } \\
\hline$A^{0}\left(10^{i} 20 \mathrm{~cm}^{3} \operatorname{molec}^{i^{1}} \sec ^{i^{1}}\right)$ & $4: 27 £ 10^{i} 4$ & $6: 19$ & 6:93 \\
\hline $\mathrm{n}$ & $3: 85$ & $2: 62$ & $2: 60$ \\
\hline$B(\mathrm{~K})$ & 1617 & 2440 & 2454 \\
\hline \multicolumn{4}{|c|}{ KSG surface } \\
\hline$A^{0}\left(10^{i}{ }^{16} \mathrm{~cm}^{3} \mathrm{molec}^{i^{1}} \mathrm{sec}^{{ }^{1}}\right)$ & $0: 145$ & $1: 84$ & $1: 79$ \\
\hline $\mathrm{n}$ & $1: 89$ & 1:56 & $1: 57$ \\
\hline $\mathrm{B}(\mathrm{K})$ & 2386 & 2601 & 2600 \\
\hline \multicolumn{4}{|c|}{ Fits to experimental results } \\
\hline Reference & - & [11] & [11] \\
\hline $\mathrm{A}^{0}\left(10^{\mathrm{i} 21} \mathrm{~cm}^{3} \mathrm{molec}^{\mathrm{i}^{1}} \mathrm{sec}^{\mathrm{i}^{1}}\right)$ & - & $0: 748$ & $5: 6$ \\
\hline $\mathrm{n}$ & - & $3: 13$ & $2: 87$ \\
\hline$B(\mathrm{~K})$ & - & 1550 & 1766 \\
\hline
\end{tabular}

\begin{tabular}{|c|c|c|c|c|c|c|}
\hline \multicolumn{7}{|c|}{ Table IV. Comparison of rate coefficients computed from fits. } \\
\hline \multicolumn{7}{|c|}{ Two-parameter fits } \\
\hline \multicolumn{7}{|c|}{ (The rate coefficients are in units of $10^{i} 16 \mathrm{~cm}^{3}$ molecule ${ }^{1} \sec ^{i^{1}}$ ) } \\
\hline$T_{3}$ & S1 & S1A & S2 & KSG & Expt. & Ref. \\
\hline 326 & $0: 59$ & 8:58 & $1: 41$ & $5: 42$ & $3: 11$ & [3] \\
\hline 352 & $1: 48$ & 2:05 & 3:09 & $11: 37$ & $5: 40$ & [5] \\
\hline 416 & 9:80 & $12: 93$ & $17: 62$ & $52: 04$ & $36: 39$ & [7] \\
\hline " $(\%)$ & $76: 5$ & $66: 1$ & 49:5 & $70: 7$ & - & - \\
\hline \multicolumn{7}{|c|}{ Three-parameter fits } \\
\hline \multicolumn{7}{|c|}{ (The rate coefficients are in units of $10^{i}{ }^{14} \mathrm{~cm}^{3}$ molecule ${ }^{1} \mathrm{sec}^{\mathrm{i}}{ }^{1}$ ) } \\
\hline 567 & $1: 22$ & $1: 39$ & $1: 37$ & $3: 70$ & $2: 02$ & [11] \\
\hline 570 & $1: 24$ & $1: 42$ & $1: 37$ & $3: 97$ & $2: 05$ & {$[11]$} \\
\hline${ }^{\prime \prime}(\%)$ & $42: 8$ & $33: 8$ & $34: 9$ & $87: 5$ & - & - \\
\hline
\end{tabular}




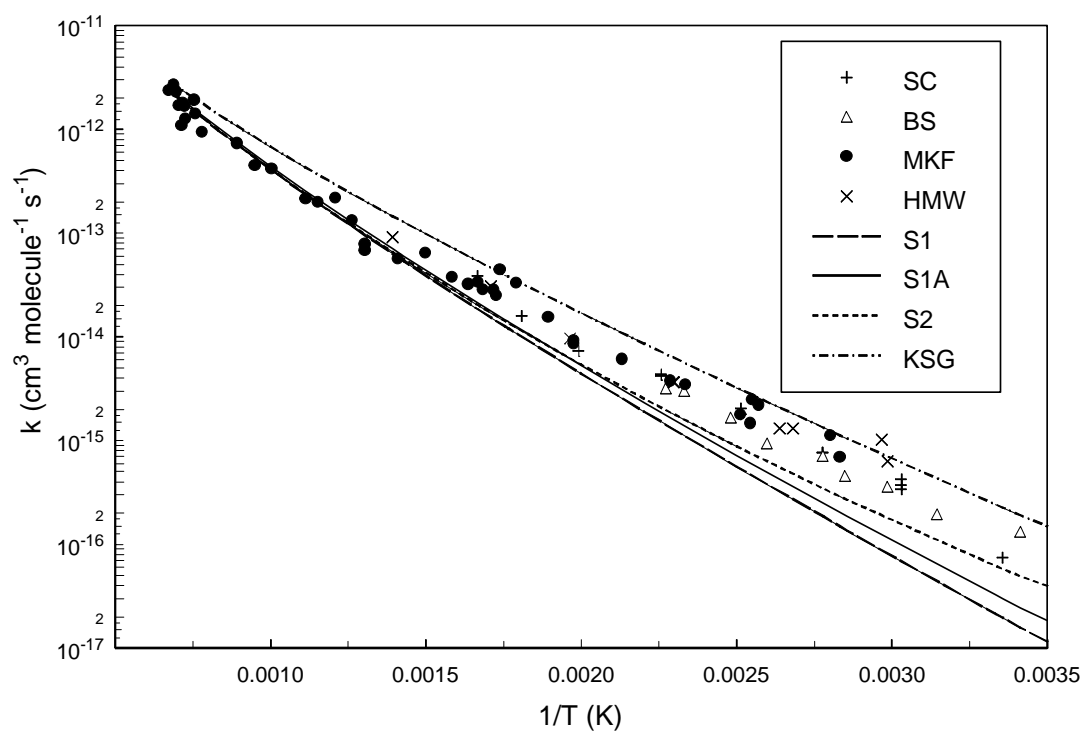

Figure 1: Comparison of the ICVT/ ${ }^{1}$ OMT (Morse I) thermal rate coefficients computed for the three RSW surfaces and the KSG surface with experimental data. The experimental results shown are taken from Refs. [3] (SC), [5] (BS), [7] (HMW), and [11] (MKF). 